\title{
Middle Cranial Fossa Repair of Temporal Bone Spontaneous CSF Leaks With Hydroxyapatite Bone Cement
}

\author{
Mohamedkazim M. Alwani, MD ㅇ; Mohamad Z. Saltagi, MD; Margaret E. MacPhail, MS; \\ Rick F. Nelson, MD, PhD (D)
}

\begin{abstract}
Objectives: To determine the safety and effectiveness of the middle cranial fossa (MCF) approach in repairing spontaneous cerebrospinal fluid (sCSF) leaks.

Study Design: Retrospective cohort study.

Methods: Patient with sCSF leaks repaired by MCF approach between January 1, 2014 and August 31, 2019 were included. Demographic information, clinical and surgical findings, and postoperative outcomes were recorded.

Results: The cohort $(n=45)$ included 24 tegmen repairs by multilayer reconstruction using hydroxyapatite cement and 21 cases of multilayer repair without hydroxyapatite cement. Ten MCF repairs were performed on patients $\geq 65$ years old. Twenty (53\%) ears had multiple tegmen defects (range, 1-9 tegmen defects) and 78\% of patients had $\geq 1$ encephaloceles. All sCSF leaks were resolved with one surgical intervention. There were no major intracranial complications. Transient expressive aphasia occurred in 2 patients. Medical complications occurred in four patients. There were no short-term postoperative CSF leaks with bone cement reconstruction and two postoperative leaks without bone cement. One resolved with lumbar drain (LD) and the other resolved without treatment. The average (SD) length of stay (LOS) with bone cement was shorter than in patients without bone cement $(2.54$ [0.83] days vs. 3.52 [1.99] days, $P<.05)$. There have been no long-term CSF leak recurrences with an average (SD) follow-up of 13.5 (12.9) months (range 0.25-46 months).

Conclusions: MCF approach for sCSF repairs demonstrate efficacious outcomes, particularly with tegmen reconstruction using hydroxyapatite cement. The approach exhibited no serious adverse events and few complications requiring intervention. Therefore, MCF is a safe and effective approach to resolve sCSF leaks.

Key Words: Cerebrospinal fluid leak, middle fossa craniotomy, encephalocele, obesity, complications, obstructive sleep apnea, intracranial hypertension.

Level of Evidence: 3
\end{abstract}

Laryngoscope, 00:1-9, 2020

\section{INTRODUCTION}

Spontaneous cerebrospinal fluid leaks (sCSF-L) are becoming increasingly common. ${ }^{1}$ They occur secondary to lateral or anterior skull base bony dehiscence in addition to dural defects, which are often associated with encephaloceles. Clinically, lateral leaks in the temporal bone cause conductive hearing loss (CHL) and persistent ear effusions with or without chronic clear otorrhea and/or rhinorrhea, while anterior skull base leaks can lead to chronic clear rhinorrhea. sCSF-L is understood to be a spontaneous disease process that occurs without an inciting incident of trauma, prior skull base surgery, or intracranial tumor. ${ }^{2}$ SCSF-L are most prevalent in middle-aged, obese females. ${ }^{3-5}$ Recent studies highlight the role of obesityrelated factors such as elevated intracranial pressure

Additional supporting information may be found in the online version of this article.

From the Department of Otolaryngology, Head and Neck Surgery (M.M.A., M.Z.s., R.F.N.), Indiana University School of Medicine, Indianapolis, Indiana, U.S.A.; Indiana University School of Medicine (M.E.M., R.F.N.), Indianapolis, Indiana, U.S.A.; and the Department of Neurosurgery (R.F.N.), Indiana University School of Medicine, Indianapolis, Indiana, U.S.A.

Editor's Note: This Manuscript was accepted for publication on April 30, 2020.

The authors have no funding or conflicts of interest to declare.

Send correspondence to Rick F. Nelson, MD, PhD, Indiana University College of Medicine, 980 W. Walnut Street, WH426C, Indianapolis, IN 46202m. E-mail: ricnelso@iupui.edu

DOI: 10.1002/lary.28761
(ICP) and obstructive sleep apnea (OSA) in development of sCSF-L. ${ }^{1,4,6-11}$ The rate of SCSF-L and their surgical repair, continue to increase over recent decades, mirroring the increasing obesity during this time period. .,4,12,13 $^{1}$

Nearly all temporal bone sCSF-L occur through the tegmen (tympani or mastoideum) and through the temporal lobe dura. Posterior fossa plate leaks over the cerebellum are rare. ${ }^{14}$ Surgical approaches to repair temporal bone sCSF-L include middle cranial fossa (MCF) craniotomy, transmastoid and combined MCF/transmastoid approaches. ${ }^{4}$ Repair of SCSF-L is indicated to reduce the risk of meningitis, stop otorrhea and improve conductive hearing. Advantages of MCF approach include broad exposure to the entire tegmen and skull base, but risks of the craniotomy have been discussed as reasons to avoid MCF approach. Postoperative CSF leak rates vary by surgical approach and case series, ranging from $0 \%$ to $22 \%{ }^{4}$ This study evaluates one of the largest cohorts of SCSF-L repaired exclusively by the MCF approach to determine the safety and effectiveness of the repair with and without the use of hydroxyapatite cement.

\section{METHODS}

\section{Study Approval}

After obtaining institutional review board approval (\#1907071217), the electronic medical record database was 


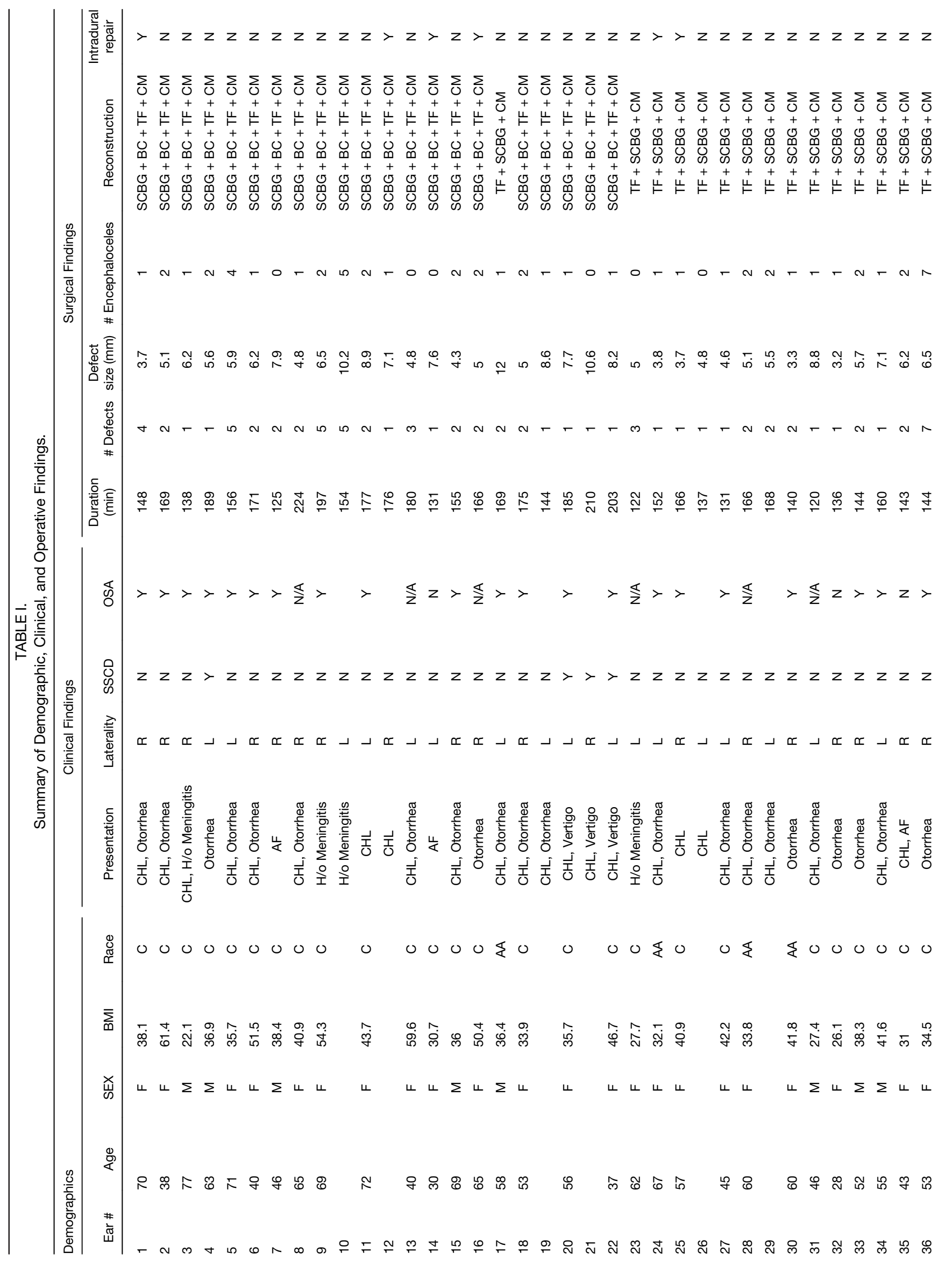




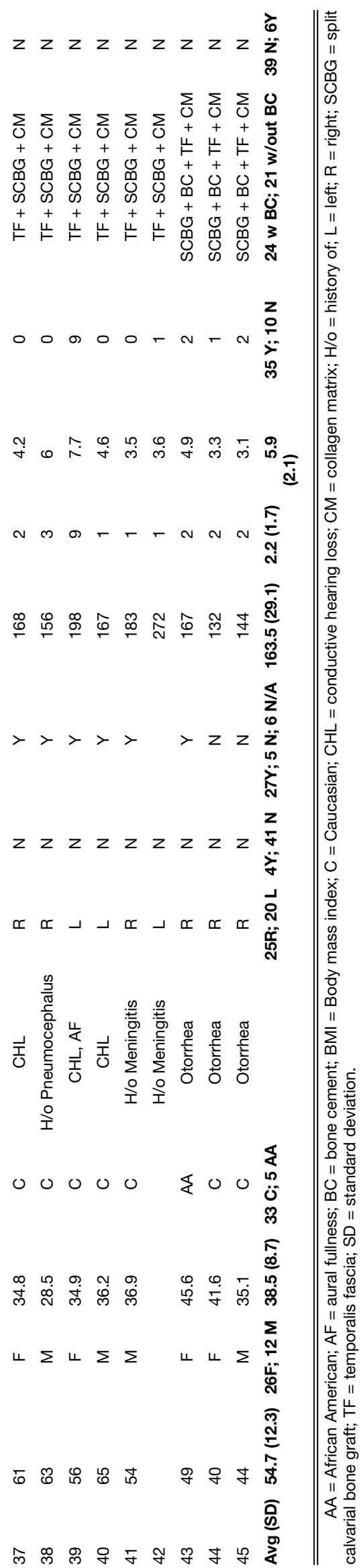

searched for all patients who underwent MCF approach for repair of SCSF-L by the senior author (R.N.) between January 1, 2014 and August 31, 2019.

\section{Eligibility Criteria and Patient Selection}

All patients who received MCF approach for sCSF-L were included. Patients with cholesteatoma, neoplasms, history of temporal bone trauma, and prior skull base surgery were excluded from the study. Patients with non-serviceable hearing and CSF leak were excluded and treated with subtotal petrousectomy. sCSF-L at our institution are exclusively repaired using MCF approach. Diagnosis of SCSF-L was made as follows: 1) otomicroscopic evidence of clear middle ear fluid or clear pulsatile fluid emerging from pressure equalization tube in the absence of cholesteatoma and 2) evidence of tegmen defects $(>3 \mathrm{~mm})$ on coronal sections of highresolution temporal bone CT scans. The ruler function of the Synapse PACS Imaging System (Fujifilm, Tokyo, Japan) was used to measure the main skull base defect on coronal computed tomography (CT) scan sections in millimeters to the decimal place. Beta-2 transferrin testing, T2-weighted magnetic resonance imaging (MRI), and/or CT/MRI cisternograms were only utilized for diagnosis of SCSF-L when diagnosis was equivocal. Demographic data, clinical findings, operative details and postoperative complications were recorded for patients who met inclusion criteria.

\section{Cisternogram Protocol}

Equivocal cases were evaluated with CT and MRI cisternograms by placing a mixture of $10 \mathrm{~mL}$ Isovue- $300 \mathrm{M}$ (CT contrast) and $0.5 \mathrm{~mL}$ MultiHance (MRI contrast) intrathecally after lumbar puncture (LP). The patients remained with their head down until contrast was seen in the upper cervical levels on fluoroscopic imaging. The patients then underwent high-resolution CT imaging followed by MRI to detect contrast egress into the middle ear or mastoid.

\section{Surgical and Reconstructive Technique}

Surgical steps of the MCF approach have been previously well described..$^{15,16}$ We administer intraoperative mannitol $(0.5 \mathrm{~g} / \mathrm{kg})$ and hyperventilate the patient to achieve an end-tidal $\mathrm{CO}_{2}$ of $<30 \mathrm{mmHg}$ to facilitate temporal lobe relaxation. Lumbar drains (LD) are not used intraoperatively per findings of prior studies. ${ }^{15}$

Previously, we used a multi-layer reconstruction technique by draping temporalis fascia over tegmen defects as a soft tissue seal, followed by placement of split calvarial bone graft (SCBG) harvested from the craniotomy. Extradural repair included the use of the collagen matrix Durepair (Medtronic, Minneapolis, $\mathrm{MN}$ ) and DuraGen (Integra LifeSciences, Plainsboro, NJ).

Using a modified multi-layer reconstruction technique, we now place a SCBG larger than the tegmen defect directly over the defect followed by HydroSet calcium phosphate bone substitute (Stryker, Kalamazoo, MI) to seal the SCBG in place (Video S1). HydroSet hardens within 8 minutes and forms hydroxyapatite. Subsequently, the entire tegmen is covered with bone cement followed by multilayer extra-dural repair as described above. If a superior semicircular canal dehiscence (SSCD) is encountered, it is addressed prior to tegmen reconstruction by plugging the defect with bone wax.

In the event that a lateral dural defect is encountered after raising the craniotomy bone flap, the defect is repaired intradurally with Durepair, which is oversewn with 4.0 Nurolon suture (Johnson \& Johnson, New Brunswick, NJ) prior to temporal lobe elevation. 


\section{Statistical Analysis}

Student unpaired t-test comparison of means with $P<.05$ considered statistically significant.

\section{RESULTS}

\section{Demographics and Surgical Findings}

Between January 2014 and August 2019, 45 MCF operations for sCSF-L were performed (Table I). Seven patients underwent bilateral repair for a total of 38 patients. Mean (standard deviation [SD]) age was 54.7 (12.3) years (range, 28-77 years). MCF repairs were performed on 10 patients $\geq 65$ years old. Twenty-six $(68.4 \%)$ patients were female. Average (SD) BMI was 38.5 (8.7) $\mathrm{kg} / \mathrm{m}^{2}$ (range, $26.1-61.4 \mathrm{~kg} / \mathrm{m}^{2}$ ). Five (13\%) patients were African American and all others were Caucasian. Four of 45 ears also had SSCD at time of presentation. Five (13\%) patients had a history of meningitis prior to diagnosis. Table I summarizes demographic and clinical findings.

\section{Diagnosis of Temporal Spontaneous CSF Leak}

A diagnostic algorithm for ${ }^{2} \mathrm{CSF}-\mathrm{L}$ is outlined in Figure 1A. All patients with temporal sCSF-L were evaluated using clinical exam and high-resolution CT imaging to evaluate for tegmen defects (Fig. 1B,C). Given that the mean (SD) tegmen defect size in our cohort was 5.9 (2.1) $\mathrm{mm}$ (range, 3.1-10.6 mm), we determined a defect size of $3.0 \mathrm{~mm}$ to be an appropriate cut-off point in our algorithm. Many patients exhibited clear pulsatile fluid from a pressure equalizing tube that increased with valsalva. Select patients had sampling of the fluid for Beta-2 transferrin testing or a diagnostic cisternogram (Fig. 1D).

\section{Operative Repair}

All temporal bone sCSF-L were repaired with MCF craniotomy. Intraoperatively, 20 (53\%) ears had more than one tegmen defect (range, 1-9 tegmen defects). All cases of concurrent superior semicircular canal dehiscence (SSCD, Fig. 2A) were repaired at the time of CSF leak repair. SSCD and tegmen defects were exposed after careful elevation of the temporal lobe without suctioning near the dehiscence (Fig. 2B). All cases of SSCD were repaired with bone wax occlusion. Tegmen defects were repaired with SCBG

A

Persistent middle ear fluid
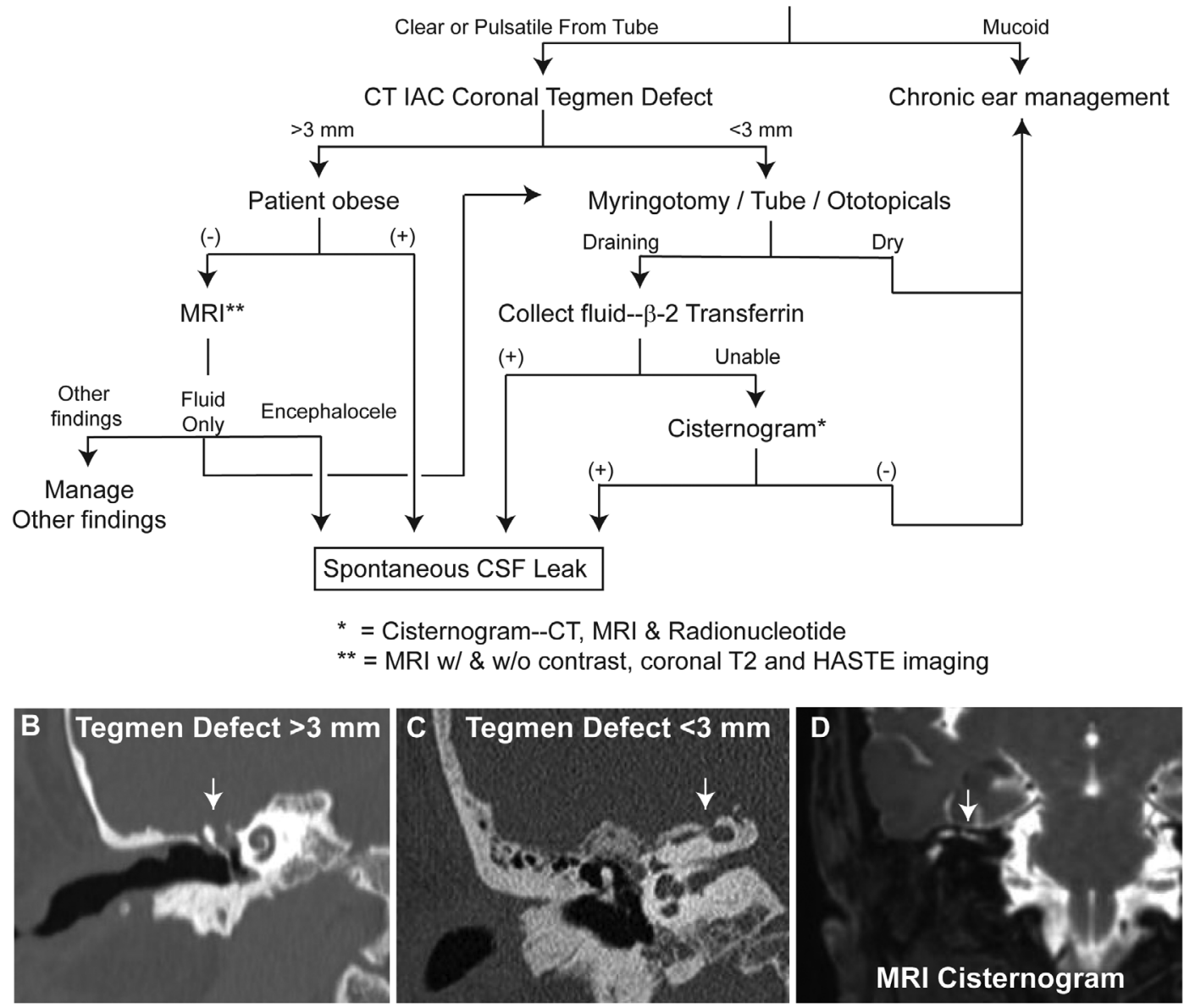

Fig. 1. Diagnosis of temporal spontaneous CSF leaks. (A) Diagnostic algorithm including at least clinical exam and high resolution CT imaging. (B) Example of coronal CT image of a large $(>3 \mathrm{~mm}$ ) tegmen defect and sCSF-L. Note the calvarial thinning. (C) Example of coronal CT image of a small tegmen defect $(<3 \mathrm{~mm})$ in a patient who had a diagnostic MRI cisternogram (D) that confirmed CSF leak. CSF = cerebrospinal fluid; $\mathrm{CT}$ = computed tomography; $\mathrm{MRI}=$ magnetic resonance imaging; sCSF-L = spontaneous cerebrospinal fluid leak. 

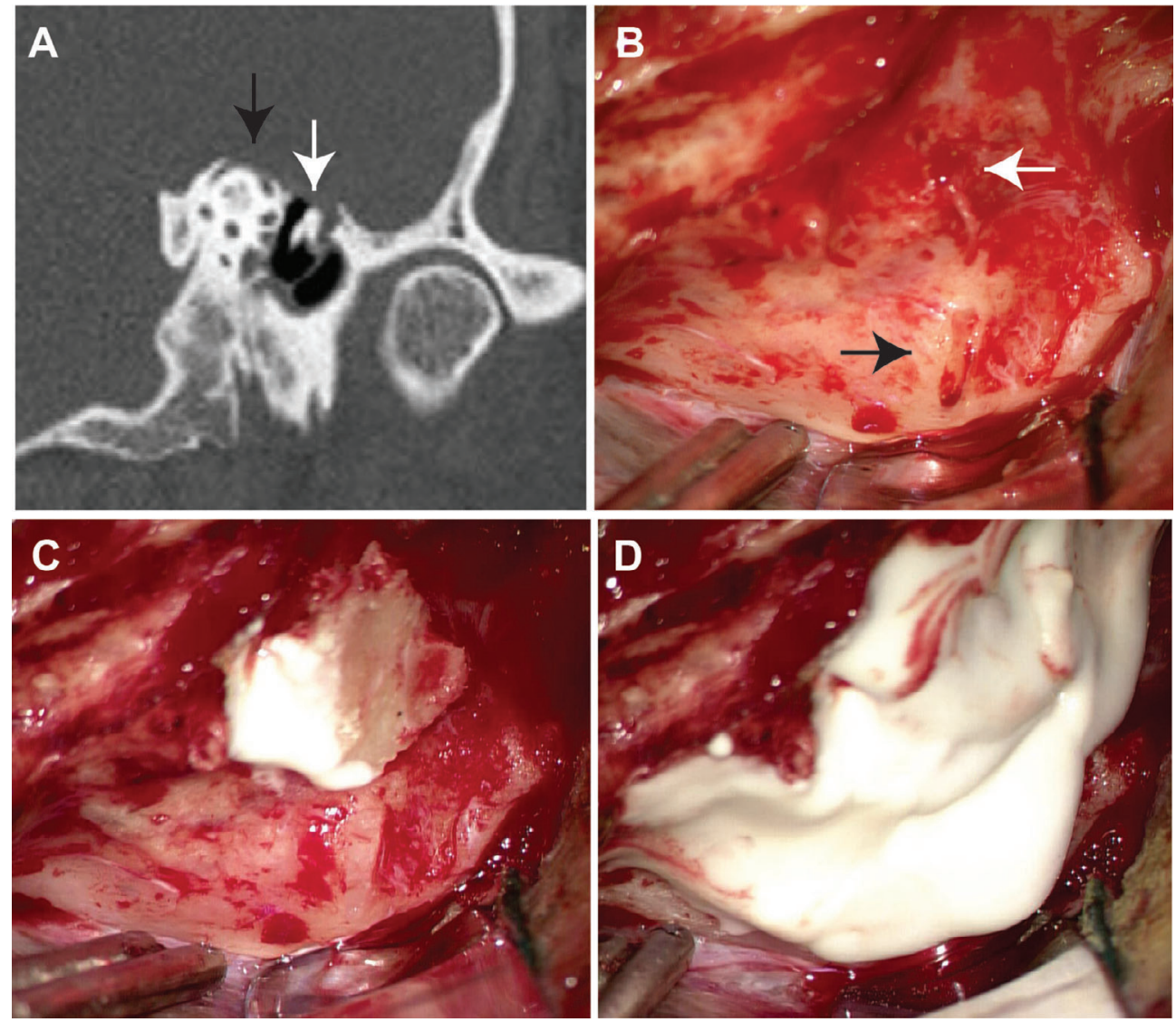

Fig. 2. Operative repair of concurrent SSCD and SCSF-L. (A) Example of CT Pöschl view of left ear demonstrating SSCD (black arrow) and large tegmen defect (white arrow). (B) Intraoperative view of same patient demonstrating open SSCD (black arrow) and encephalocele in tegmen defect (white arrow). (C) Intraoperative view after wax occlusion of SSCD and SCBG with bone cement over tegmen defect. (D) Skull base reconstruction with bone cement. CT = computed tomography; SCSF-L = spontaneous cerebrospinal fluid leak; SCBG = split calvarial bone; SSCD = superior semicircular canal dehiscence. [Color figure can be viewed in the online issue, which is available at www.laryngoscope.com.]

(Fig. 2C) followed by sealing the entire skull base with hydroxyapatite bone cement (Fig. 2D).

Encephaloceles were encountered in $78 \%$ of cases (Table I). Removal of encephaloceles was achieved in all cases without mastoidectomy and without injury to the ossicles or facial nerve. Early in the series, multilayer reconstruction was performed with fascia, SCBG and Durepair. Addition of hydroxyapatite bone cement is currently used in all cases to reinforce the tegmen and the entire middle fossa floor, prior to extradural repair. (Fig. 2C,D; Video 1)
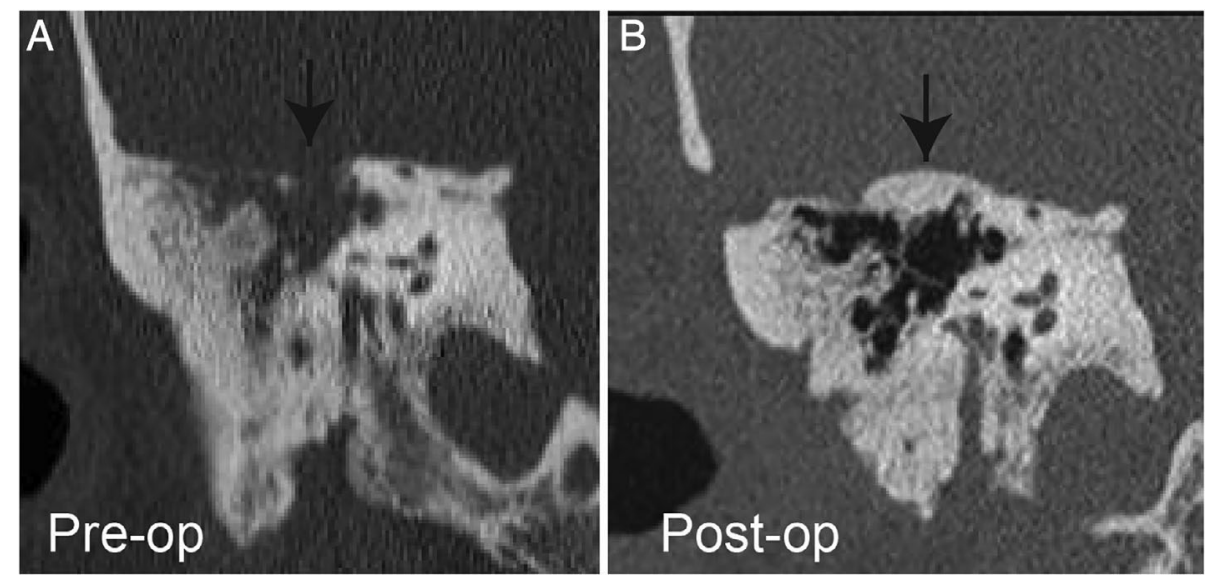

Fig. 3. Skull base reconstruction with bone cement. (A) Preoperative coronal CT demonstrating large tegmen mastoidium defect (black arrow) with fluid in the middle ear and mastoid. (B) One-month postoperative coronal CT image demonstrating skull base reconstruction with bone cement (black arrow) with an aerated middle ear and mastoid. CT = computed tomography. 
TABLE II.

Summary of Postoperative Course and Complications.

\begin{tabular}{|c|c|c|c|c|c|c|}
\hline \multirow[b]{2}{*}{ Ear \# } & \multicolumn{2}{|c|}{ Postoperative Course } & \multicolumn{4}{|c|}{ Postoperative Complications } \\
\hline & Total LOS (d) & FU Length (m) & HB Grade & Otorrhea & Rhinorrhea & Meningitis \\
\hline 1 & 2 & 2 & 1 & $\mathrm{~N}$ & $\mathrm{~N}$ & $\mathrm{~N}$ \\
\hline 3 & 2 & 0.5 & 1 & $\mathrm{~N}$ & $\mathrm{~N}$ & $\mathrm{~N}$ \\
\hline 4 & 2 & 1 & 1 & $\mathrm{~N}$ & $\mathrm{~N}$ & $\mathrm{~N}$ \\
\hline 7 & 2 & 4 & 1 & $\mathrm{~N}$ & $\mathrm{~N}$ & $\mathrm{~N}$ \\
\hline 8 & 2 & 8 & 1 & $\mathrm{~N}$ & $\mathrm{~N}$ & $\mathrm{~N}$ \\
\hline 9 & 4 & 9 & 1 & $\mathrm{~N}$ & $\mathrm{~N}$ & $\mathrm{~N}$ \\
\hline 10 & 2 & 7 & 1 & $\mathrm{~N}$ & $\mathrm{~N}$ & $\mathrm{~N}$ \\
\hline 11 & 3 & 12 & 1 & $\mathrm{~N}$ & $\mathrm{~N}$ & $\mathrm{~N}$ \\
\hline 15 & 2 & 14 & 1 & $\mathrm{~N}$ & $\mathrm{~N}$ & $\mathrm{~N}$ \\
\hline 16 & 2 & 9.5 & 1 & $\mathrm{~N}$ & $\mathrm{~N}$ & $\mathrm{~N}$ \\
\hline 17 & 3 & 13 & 1 & $\mathrm{~N}$ & $\mathrm{~N}$ & $\mathrm{~N}$ \\
\hline 18 & 4 & 18 & 1 & $\mathrm{~N}$ & $\mathrm{~N}$ & $\mathrm{~N}$ \\
\hline 19 & 3 & 13 & 1 & $\mathrm{~N}$ & $\mathrm{~N}$ & $\mathrm{~N}$ \\
\hline 20 & 4 & 12 & 1 & $\mathrm{~N}$ & $\mathrm{~N}$ & $\mathrm{~N}$ \\
\hline 21 & 2 & 9 & 1 & $\mathrm{~N}$ & $\mathrm{~N}$ & $\mathrm{~N}$ \\
\hline 22 & 4 & 17 & 1 & $\mathrm{~N}$ & $\mathrm{~N}$ & $\mathrm{~N}$ \\
\hline 23 & 2 & 18 & 1 & $\mathrm{~N}$ & $\mathrm{~N}$ & $\mathrm{~N}$ \\
\hline 24 & 3 & 22 & 1 & $\mathrm{~N}$ & $\mathrm{~N}$ & $\mathrm{~N}$ \\
\hline 32 & 3 & 14 & 1 & $\mathrm{~N}$ & $\mathrm{~N}$ & $\mathrm{~N}$ \\
\hline 33 & 4 & 20.5 & 1 & $\mathrm{~N}$ & $\mathrm{~N}$ & $\mathrm{~N}$ \\
\hline 34 & 7 & 19.5 & 1 & $\mathrm{~N}$ & $\mathrm{~N}$ & $\mathrm{~N}$ \\
\hline 35 & 9 & 33 & 1 & $Y$ & $N$ & $\mathrm{~N}$ \\
\hline 36 & 3 & 40 & 1 & $\mathrm{~N}$ & $Y$ & $\mathrm{~N}$ \\
\hline 37 & 3 & 33 & 1 & $\mathrm{~N}$ & $\mathrm{~N}$ & $\mathrm{~N}$ \\
\hline 38 & 3 & 10 & 1 & $\mathrm{~N}$ & $\mathrm{~N}$ & $\mathrm{~N}$ \\
\hline 39 & 2 & 37 & 1 & $\mathrm{~N}$ & $\mathrm{~N}$ & $\mathrm{~N}$ \\
\hline 40 & 3 & 44.5 & 1 & $\mathrm{~N}$ & $\mathrm{~N}$ & $\mathrm{~N}$ \\
\hline 41 & 7 & 46 & 1 & $\mathrm{~N}$ & $\mathrm{~N}$ & $\mathrm{~N}$ \\
\hline 42 & 2 & 41 & 1 & $\mathrm{~N}$ & $\mathrm{~N}$ & $\mathrm{~N}$ \\
\hline 43 & 2 & 0.25 & 1 & $\mathrm{~N}$ & $\mathrm{~N}$ & $\mathrm{~N}$ \\
\hline 44 & 2 & 0.25 & 1 & $\mathrm{~N}$ & $\mathrm{~N}$ & $\mathrm{~N}$ \\
\hline 45 & 2 & 0.25 & 1 & $\mathrm{~N}$ & $N$ & $\mathrm{~N}$ \\
\hline Avg (SD) & $3.0(1.6)$ & 13.5 (12.9) & 45 HB 1 & $44 \mathrm{~N} ; 1 \mathrm{YY}$ & $44 \mathrm{~N} ; 1 \mathrm{Y}$ & $45 \mathrm{~N}$; OY \\
\hline
\end{tabular}

$\mathrm{FU}=$ follow up; $\mathrm{HB}$ = House Brackmann; LOS = length of stay; $\mathrm{N}=$ no; SD = Standard deviation; $\mathrm{Y}$ = yes.

Postoperative imaging after bone cement reconstruction demonstrates skull base reconstruction that resembles bone and resolution of the sCSF-L demonstrated by an aerated middle ear and mastoid (Fig. 3A,B). Average (SD) operative time without bone cement was not significantly different than with bone cement (167 [26] minutes 
vs. 159 [32] minutes, $P=.36$ ). However, average (SD) length of stay (LOS) for patients repaired with bone cement was shorter than those who were not (2.54 [0.83] days vs. 3.52 [1.99] days, $P=.03$ ).

The lateral temporal lobe dura required repair after craniotomy in 6 patients, four of whom were $\geq 65$ years old. Subsequently, CSF leak repair was performed extradurally in a multilayer fashion in all cases.

\section{Complications and CSF Leak Recurrence}

No patients experienced postoperative meningitis, wound infections, seizure, epidural hematoma, or need for reoperation. Four medical complications occurred in the perioperative period, including ileus, heart block masking atrial fibrillation, diabetic nephropathy, and a case of metabolic encephalopathy from hyperglycemia requiring readmission.

Two patients (ear 10 and 34 in Table I) experienced expressive aphasia that resolved with postoperative steroids. Both cases had left-sided surgery. Another patient required a tympanostomy tube for eustachian tube dysfunction, which resulted in a dry ear.

Two patients presented with early postoperative CSF leaks. Both patients did not have bone cement reconstructions. One patient had postoperative otorrhea that resolved with LD placement for 5 days. Another patient experienced clear rhinorrhea after discharge which resolved within 3 weeks without intervention, as demonstrated by exam and imaging. This patient ultimately was found to have underlying elevated ICP and underwent CSF diversion with a ventriculoperitoneal shunt. No other patients underwent intraoperative or postoperative LD placement.

There are no long-term CSF leak recurrences with an average (SD) follow-up of 13.5 (12.9) months (range, 0.25-46 months). Table II summarizes the postoperative course and complications.

\section{Postoperative Management}

All patients were advised to undergo LP for ICP measurement after surgical repair. Eleven patients underwent

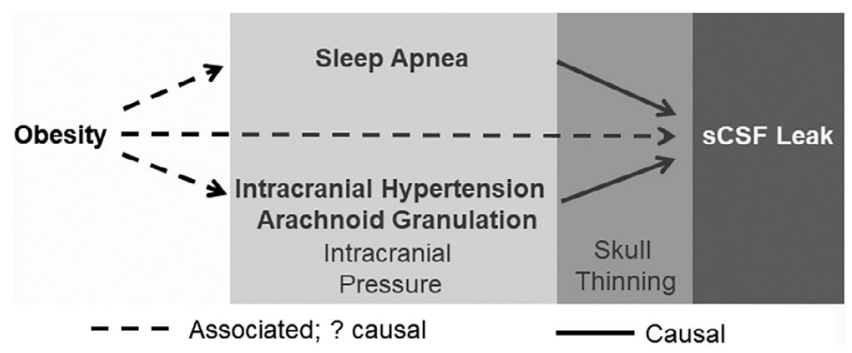

Fig. 4. Pathophysiology of SCSF-L. Obesity is highly associated with SCSF-L, but obesity is likely not causal. Obesity is highly associated with conditions that transiently (OSA) or chronically (intracranial hypertension) elevate intracranial pressure. Both OSA and intracranial hypertension independently thin the skull and skull base likely leading to SCSF-L development. OSA = obstructive sleep apnea; sCSF-L = spontaneously cerebrospinal leak.
LP with average opening pressure of $23 \mathrm{mmHg}$ (range, 6-40 mmHg). All patients were advised to undergo a formal polysomnogram for OSA testing; Twenty-seven (71\%) patients had OSA, five did not have OSA, and six declined this testing (Table I).

\section{DISCUSSION}

The MCF approach is a safe, definitive method of repairing sCSF-L. Comparatively, transmastoid and/or mini-craniotomy approaches offer a highly successful and low-morbidity method of repairing sCSF-L, ${ }^{17}$ but the MCF approach confers great benefit in the patient with multiple tegmen defects ( $>50 \%$ of our cohort) given its wide exposure of the entire tegmen. Our patients experienced no cases of meningitis, surgical site infections, or major intracranial complications. Transient complications secondary to prior comorbidities and transient expressive aphasia were the only complications observed. The two patients who developed brief postoperative CSF leaks did not have bone cement reconstruction. Our practice has since evolved to incorporate bone cement into all repairs.

The incidence of sCSF leaks is increasing over time. Our patient cohort mirrors previous studies demonstrating that SCSF-L are most prevalent in middle-aged (mean 45-65 years), obese (average body mass index [BMI] = 35-38$\mathrm{kg} / \mathrm{m}^{2}$ ) females (female:male ratio $=70: 30$ ).$^{3-5}$ Previous studies also highlight key comorbid conditions associated with obesity and SCSF-L, including elevated ICP and OSA. ${ }^{1,4,6-11}$ However, the pathophysiology of SCSF-L continues to be investigated. ${ }^{1,4,12,13}$

\section{Role of Obesity in SCSF-L}

Literature has demonstrated that most patients who undergo sCSF-L repair are obese ${ }^{1,8,10,13,14,18,19}$ (Fig. 4). Despite the high prevalence of obesity among sCSF-L patients, obesity alone is not directly responsible for skull thinning (Fig. 4). Comparison of calvarial thickness (squamous temporal bone) among age-matched obese and non-obese patients undergoing cochlear implantation found calvarial thickness was not impacted by BMI. ${ }^{3}$ However, sCSF-L patients have $23 \%$ thinner calvarium when compared to age- and BMI-matched controls. ${ }^{3}$ Since the extracranial zygoma thickness is unaffected in sCSF-L patients, ${ }^{3}$ sCSF-L likely occur due to an intracranial process that causes isolated skull thinning independent of obesity. ${ }^{3}$ Researchers hypothesize obesity-related factors, such as idiopathic intracranial hypertension (IIH) and OSA, play a role in the intracranial process that results in the development of sCSF-L $\mathrm{L}^{3,4,20-22}$ (Fig. 4).

\section{Role of Intracranial Pressure in sCSF-L}

Increased ICP, whether transient or chronic, likely contributes to development of sCSF-L, through skull base thinning and subsequent dural exposure and tearing. ${ }^{20-24}$ Pre-operative ICP measurements in anterior skull base sCSF-L patients show elevated mean opening pressure of $33.0 \mathrm{~cm} \mathrm{H}{ }_{2} \mathrm{O} .^{25}$ Elevated ICP has been observed in $10 \%$ to $66 \%$ of patients presenting for evaluation of $\mathrm{sCSF}-\mathrm{L}$ 
based on LP. ${ }^{6,11,26}$ Because ICP is known to vary throughout the day and with positioning, LP readings may also vary within the same patient.

Evaluating the relationship between calvarial thickness and opening ICP on LP showed calvarium thickness increases with age among patients with normal opening pressure, but decreases with aging in those with high ICP. ${ }^{22}$ In addition, high ICP patients had significantly decreased squamous temporal bone and skull base thickness compared with normal ICP patients. ${ }^{22}$ These data implicate elevated ICP as a direct cause of skull thinning (Fig. 4). Despite these findings, this study was limited by a single-time point ICP measurement. sCSF-L patients may not all have chronically elevated ICP; transient spikes in ICP may also be implicated.

\section{Role of OSA in SCSF-L}

OSA has a strong association with obesity ${ }^{27,28}$ and is known to cause transient intracranial hypertension during apneic episodes ${ }^{29,30}$ (Fig. 4). OSA is present in $83.3 \%$ of $\mathrm{SCSF}-\mathrm{L}$ patients, ${ }^{21}$ much higher than previously reported studies. ${ }^{1,7,31,32}$

Moderate-severe OSA patients have approximately $10 \%$ thinner calvaria and $14 \%$ thinner skull bases than non-OSA patients. ${ }^{20}$ Extracranial zygoma thickness was not different between groups. In addition, the tegmen mastoideum was dehiscent in nearly twice as many OSA patients. $^{20}$ This implicates OSA as a direct cause of skull thinning (Fig. 4).

We hypothesize that OSA, on a chronic basis, causes progressive thinning of the skull base through its transient effects on ICP during apneic episodes. Apnea elicits transient hypercarbia, resulting in cerebral vasodilation and impaired venous drainage, and subsequent spikes in ICP. ${ }^{29,30}$ Transiently or chronically elevated ICP in these patients can cause progressive thinning which can lead to sCSF-L, particularly in inherently thin areas of skull base such as the tegmen and cribriform plate. Erosion of bone to a magnitude of $1 \mathrm{~mm}$ can lead to CSF otorrhea and/or rhinorrhea ${ }^{1}$ (Fig. 4).

\section{Role of Arachnoid Granulations in SCSF-L}

Arachnoid granulations (AGs) arise from erosive pulsations of arachnoid villi in close proximity to thin portions of middle fossa and posterior fossa dura and underlying skull base and are implicated in $\mathrm{sCSF}_{-} \mathrm{L}^{33}$ (Fig. 4). AGs occur most frequently in the middle fossa and often cause larger defects when compared to posterior fossa. ${ }^{33,34}$ Defects can occur independent of obesity and have been found to occur more frequently with advancing age. ${ }^{33}$ A review of temporal bone specimens found that AGs fully penetrated dura and made contact with cortical surfaces in $13 \%$ of donor temporal bones. ${ }^{33}$

\section{Use of Hydroxyapatite Bone Cement}

The MCF approach allows access to the entire skull base in a patient population that typically has more than one tegmen defect. We exclusively use hydroxyapatite bone cement for definitive skull base repair with no observed immediate or long-term CSF leaks. Additionally, bone cement provides long-term reinforcement of the skull base in a patient population with ongoing skull base thinning. With such a robust skull base seal, patients likely can resume continuous positive airway pressure (CPAP) use for OSA early after surgery with potentially less pneumocephalus risk. While we do not have data to support a specific timeframe for allowing patients to resume CPAP, we anecdotally report than one of our patients self-resumed CPAP 5 days postoperatively and did not develop pneumocephalus. The ability to potentially resume CPAP earlier is felt to be a significant strength of using hydroxyapatite bone cement, given that over $83 \%$ of $\mathrm{sCSF}$ leak patients have OSA, ${ }^{21}$ and the mainstay of treatment for OSA in adults is CPAP. ${ }^{35-38}$

We also noted decreased LOS in these patients. Although the mechanism for decreased LOS with bone cement is not understood, this finding is important as it demonstrates the expense associated with bone cement is likely negated by a more definitive repair.

Lastly, there were no infections using bone cement. Prior studies have noted the possibility of infection when using bone cement in the mastoid, although rates of infection were relatively low. ${ }^{14,26,39}$ Specifically, Kutz et al. utilized bone cement in $82.4 \%$ of 55 patients undergoing repair of $\mathrm{SCSF}-\mathrm{L}^{14}$; one patient who underwent transmastoid repair of a posterior fossa defect developed an infection post-operatively, which resolved after a return to the OR to clear granulation tissue off of the bone cement. ${ }^{14}$ Kveton et al. utilized bone cement in repairing $13 \mathrm{sCSF}-\mathrm{L}$ through a transmastoid approach, and reported one infection in a patient who pre-operatively had chronic suppurative otitis media, concluding that this procedure should be avoided in the setting of active infection. ${ }^{39}$

Lastly, our current practice is to avoid placing bone cement over a dehiscent geniculate ganglion, so as to avoid potential nerve injury.

\section{Hearing Outcomes}

Resolution of $\mathrm{CHL}$ with $\mathrm{CSF}$ leak repair has been described. ${ }^{16}$ MCF repair of sCSF leaks demonstrates $96 \%$ of patients have closure of the air-bone gap postoperatively. ${ }^{16}$ Combined MCF/TM approach also reported improved hearing outcomes on average, but not universally, ${ }^{40}$ likely attributed to cases involving disarticulation of the incus-stapes joint for repair of tegmen tympani defects.

\section{Algorithm-Workup of Suspected Spontaneous CSF Leak}

We devised an algorithm to summarize and illustrate the workup of a suspected sCSF-L (Fig. 1). Upon presentation with clear otorrhea/effusion and CHL, we propose that providers obtain a high-resolution coronal CT scan of the temporal bone. At least one tegmen defect on CT imaging $\geq 3 \mathrm{~mm}$ in width (Fig. 1B) is sufficient for diagnosis, based on our patient cohort in which no patients had a tegmen defect $<3 \mathrm{~mm}$. In more challenging cases where tegmen defects are $<3 \mathrm{~mm}$ (Fig. 1C), confirmatory testing with 
Beta-2 transferrin or cisternogram is recommended (Fig. 1D). We propose this algorithm not as rigid instruction, but rather as an approximate clinical reference aid for initial patient evaluation, based on our approach at our high-volume, tertiary referral neurotologic practice.

\section{Study Limitations}

This study is limited by the lack of prospective longterm evaluation for leak recurrence, and some patients may have been lost to follow-up. The lack of a complication in this series does not mean that surgical complications will not occur in the future. Decreased LOS in patients with bone cement may be attributable to external reasons, such as improvement in postoperative care.

\section{CONCLUSIONS}

This series demonstrates that MCF approach for repair of temporal sCSF-L is highly effective. Use of hydroxyapatite bone cement resulted in decreased LOS and is a definitive method for skull base reconstruction. In experienced hands, the safety profile of the MCF approach is excellent in all patients, including those $\geq 65$ years old and those with comorbid conditions, and should be highly considered in the repair of almost any lateral skull base CSF leak.

\section{BIBLIOGRAPHY}

1. Nelson RF, Gantz BJ, Hansen MR. The rising incidence of spontaneous cerebrospinal fluid leaks in the United States and the association with obesity and obstructive sleep apnea. Otol Neurotol 2015;36:476-480.

2. Reddy M, Baugnon K. Imaging of cerebrospinal fluid rhinorrhea and Otorrhea. Radiol Clin North Am 2017;55:167-187.

3. Nelson RF, Hansen KR, Gantz BJ, Hansen MR. Calvarium thinning in patients with spontaneous cerebrospinal fluid leak. Otol Neurotol 2015;36: $481-485$.

4. Lobo BC, Baumanis MM, Nelson RF. Surgical repair of spontaneous cerebrospinal fluid (CSF) leaks: a systematic review. Laryngoscope Investig Otolaryngol 2017;2:215-224.

5. Vivas EX, McCall A, Raz Y, Fernandez-Miranda JC, Gardner P, Hirsch BE. ICP, BMI, surgical repair, and CSF diversion in patients presenting with spontaneous CSF Otorrhea. Otol Neurotol 2014;35:344-347.

6. Allen KP, Perez CL, Kutz JW, Gerecci D, Roland PS, Isaacson B. Elevated intracranial pressure in patients with spontaneous cerebrospinal fluid otorrhea. Laryngoscope 2014;124:251-254.

7. Fleischman GM, Ambrose EC, Rawal RB, et al. Obstructive sleep apnea in patients undergoing endoscopic surgical repair of cerebrospinal fluid rhinorrhea. Laryngoscope 2014;124:2645-2650.

8. Stucken EZ, Selesnick SH, Brown KD. The role of obesity in spontaneous temporal bone encephaloceles and CSF leak. Otol Neurotol 2012;33: 1412-1417.

9. Wang EW, Vandergrift WA 3rd, Schlosser RJ, Spontaneous CSF. Leaks. Otolaryngol Clin North Am 2011;44:845-856. vii.

10. LeVay AJ, Kveton JF. Relationship between obesity, obstructive sleep apnea, and spontaneous cerebrospinal fluid otorrhea. Laryngoscope 2008; 118:275-278

11. Brainard L, Chen DA, Aziz KM, Hillman TA. Association of benign intracranial hypertension and spontaneous encephalocele with cerebrospinal fluid leak. Otol Neurotol 2012;33:1621-1624.

12. Flegal KM, Kruszon-Moran D, Carroll MD, Fryar CD, Ogden CL. Trends in obesity among adults in the United States, 2005 to 2014. JAMA 2016;315: 2284-2291.

13. Cheng E, Grande D, Leonetti J. Management of spontaneous temporal bone cerebrospinal fluid leak: a 30-year experience. Am J Otolaryngol 2019;40: 97-100.
14. Kutz JW Jr, Johnson AK, Wick CC. Surgical management of spontaneous cerebrospinal fistulas and encephaloceles of the temporal bone. Laryngoscope 2018;128:2170-2177.

15. Nelson RF, Roche JP, Gantz BJ, Hansen MR. Middle cranial fossa (MCF) approach without the use of lumbar drain for the management of spontaneous cerebral spinal fluid (CSF) leaks. Otol Neurotol 2016;37:1625-1629.

16. Alwani M, Bandali E, Van Buren L, Yates CW, Nelson RF. Audiologic improvement following MCF approach for spontaneous cerebrospinal fluid leaks. Otol Neurotol 2019;40:1026-1033.

17. Kari E, Mattox DE. Transtemporal management of temporal bone encephaloceles and CSF leaks: review of 56 consecutive patients. Acta Otolaryngol 2011;131:391-394.

18. Berdahl JP, Fleischman D, Zaydlarova J, Stinnett S, Allingham RR, Fautsch MP. Body mass index has a linear relationship with cerebrospinal fluid pressure. Invest Ophthalmol Vis Sci 2012;53:1422-1427.

19. Quatre R, Attye A, Righini CA, et al. Spontaneous cerebrospinal fluid rhinorrhea: association with body weight and imaging data. J Neurol Surg B Skull Base 2017;78:419-424

20. Rabbani C, Saltagi MZ, Ye MJ, Patel JM, Manchanda S, Nelson RF. Association of obstructive sleep apnea with calvarial and skull base thinning. JAMA Otolaryngol Head Neck Surg 2018;144:513-518.

21. Rabbani CC, Saltagi MZ, Manchanda SK, Yates CW, Nelson RF. Prevalence of obstructive sleep apnea (OSA) in spontaneous cerebrospinal fluid (CSF) leaks: a prospective cohort study. Otol Neurotol 2018;39:e475-e480.

22. Rabbani CC, Patel JM, Nag A, et al. Association of intracranial hypertension with calvarial and skull base thinning. Otol Neurotol 2019;40: e619-e626.

23. Schlosser RJ, Woodworth BA, Wilensky EM, Grady MS, Bolger WE. Spontaneous cerebrospinal fluid leaks: a variant of benign intracranial hypertension. Ann Otol Rhinol Laryngol 2006;115:495-500.

24. Stevens SM, Rizk HG, Golnik K, et al. Idiopathic intracranial hypertension contemporary review and implications for the otolaryngologist. Laryngoscope 2018;128:248-256.

25. Martinez-Capoccioni G, Serramito-Garcia R, Martin-Bailon M, GarciaAllut A, Martin-Martin C. Spontaneous cerebrospinal fluid leaks in the anterior skull base secondary to idiopathic intracranial hypertension. Eur Arch Otorhinolaryngol 2017;274:2175-2181.

26. Kim L, Wisely CE, Dodson EE. Transmastoid approach to spontaneous temporal bone cerebrospinal fluid leaks: hearing improvement and success of repair. Otolaryngol Head Neck Surg 2014;150:472-478.

27. Lee W, Nagubadi S, Kryger MH, Mokhlesi B. Epidemiology of obstructive sleep apnea: a population-based perspective. Expert Rev Respir Med 2008; 2:349-364.

28. Li C, Ford ES, Zhao G, Croft JB, Balluz LS, Mokdad AH. Prevalence of selfreported clinically diagnosed sleep apnea according to obesity status in men and women: National Health and nutrition examination survey, 2005-2006. Prev Med 2010;51:18-23.

29. Jennum P, Borgesen SE. Intracranial pressure and obstructive sleep apnea. Chest 1989;95:279-283.

30. Sugita Y, Iijima S, Teshima Y, et al. Marked episodic elevation of cerebrospinal fluid pressure during nocturnal sleep in patients with sleep apnea hypersomnia syndrome. Electroencephalogr Clin Neurophysiol 1985;60: 214-219.

31. Bakhsheshian J, Hwang MS, Friedman M. Association between obstructive sleep apnea and spontaneous cerebrospinal fluid leaks: a systematic review and meta-analysis. JAMA Otolaryngol Head Neck Surg 2015;141: 733-738.

32. Thurtell MJ, Trotti LM, Bixler EO, et al. Obstructive sleep apnea in idiopathic intracranial hypertension: comparison with matched population data. J Neurol 2013;260:1748-1751.

33. Yew M, Dubbs B, Tong O, et al. Arachnoid granulations of the temporal bone: a histologic study of dural and osseous penetration. Otol Neurotol 2011;32:602-609

34. Remenschneider AK, Kozin ED, Curtin H, Santos F. Histopathology of idiopathic lateral skull base defects. Laryngoscope 2015;125:1798-1806.

35. Epstein LJ, Kristo D, Strollo PJ Jr, et al. Clinical guideline for the evaluation, management and long-term care of obstructive sleep apnea in adults. $J$ Clin Sleep Med 2009;5:263-276.

36. Farrell PC, Richards G. Recognition and treatment of sleep-disordered breathing: an important component of chronic disease management. J Transl Med 2017;15:114.

37. Marin JM, Agusti A, Villar I, et al. Association between treated and untreated obstructive sleep apnea and risk of hypertension. JAMA 2012; 307:2169-2176.

38. Punjabi NM, Caffo BS, Goodwin JL, et al. Sleep-disordered breathing and mortality: a prospective cohort study. PLoS Med 2009;6:e1000132.

39. Kveton JF, Goravalingappa R. Elimination of temporal bone cerebrospinal fluid otorrhea using hydroxyapatite cement. Laryngoscope 2000;110: 1655-1659.

40. Stevens SM, Crane R, Pensak ML, Samy RN. Analysis of audiometric outcomes following combined middle cranial fossa/transmastoid approaches for spontaneous cerebrospinal fluid otorrhea. Otolaryngol Head Neck Surg 2017;156:924-932. 